\title{
A COMPUTER PROOF OF A SERIES EVALUATION IN TERMS OF HARMONIC NUMBERS
}

\author{
RUSSELL LYONS, PETER PAULE, AND AXEL RIESE
}

\begin{abstract}
A fruitful interaction between a new randomized WZ procedure and other computer algebra programs is illustrated by the computer proof of a series evaluation that originates from a definite integration problem.
\end{abstract}

\section{INTRODUCTION}

Over the years, major computer algebra systems like Maple and Mathematica have significantly improved the tools that assist solving problems in connection with special functions, for instance, with respect to definite integration and summation. Nevertheless, in various cases the user desires to have assistance also in proving. In the spirit of Zeilberger's holonomic approach to special functions identities [9], for certain classes of expressions such provers have been developed recently within computer algebra systems. Moreover, in concrete applications it turns out that for several problems (to prove), one needs an interaction between various such packages. The object of this note is to illustrate this point by a two-fold sum that originates from a definite integration problem. More precisely, by using a brand new randomized WZ procedure together with other programs, we derive a computer algebra proof of the following result.

Theorem 1. For any positive integer $j$ and

$$
S(j):=\sum_{n \geq 1} \sum_{k}\left(\begin{array}{c}
2 n-1 \\
k
\end{array}\right) \frac{1}{2^{2 n-1}(2 n-1)(k-j-n+1 / 2)},
$$

one has

$$
S(j)=-\frac{2}{j} \sum_{m=1}^{j} \frac{1}{2 m-1} .
$$

Note that the right-hand side of (1) may be written as

$$
-\frac{2}{j}\left(H_{2 j}-\frac{1}{2} H_{j}\right)
$$

where

$$
H_{j}:=1+\frac{1}{2}+\cdots+\frac{1}{j}
$$

Date: July 16, 2002.

2000 Mathematics Subject Classification. 33C70, 33F10, 60G10, 68W30.

Key words and phrases. Computer algebra, hypergeometric summation, definite integration, WZ method.

The first author was supported in part by NSF grant DMS-0103897. The third author was supported by SFB grant F1305 of the Austrian FWF. 
is the $j$ th harmonic number.

Theorem 1 arose in work of the first author. Namely, in [1], estimation of the entropy of a certain process depended on evaluation of the integrals

$$
\int_{0}^{1} e^{-2 \pi i j t} \log \sin \pi t / 2 d t
$$

where $j \geq 1$ is an integer. While Maple does not find a closed form, Mathematica gives a formula for this integral that depends on polygamma functions at poles. Thus, the formula is nonsense as given to us, but one can massage the formula so that the imaginary part becomes the right-hand side of (1) divided by $-2 \pi$. On the other hand, one may also evaluate (2) as follows. In the power series

$$
\log (1-x)=-\sum_{n=0}^{\infty} \frac{x^{n}}{n}
$$

substitute $x:=\cos \pi t$ in order to write

$$
\begin{aligned}
\log \sin \pi t / 2 & =(1 / 2) \log \sin ^{2} \pi t / 2 \\
& =(1 / 2) \log ((1-\cos \pi t) / 2) \\
& =(1 / 2) \log (1-\cos \pi t)-(1 / 2) \log 2
\end{aligned}
$$

as an infinite series. Then integrate term by term. One arrives at $-S(j) /(2 \pi)$ for the imaginary part. This is not a completely satisfactory proof either of the correctness of the indefinite integration, nor of Theorem 1, since Mathematica does not provide any proof for its formula, and, indeed, it is known that definite integrals are not entirely trustworthy in computer algebra systems. Thus, the present paper provides one way to prove that the imaginary part of the integral (2) is equal to the right-hand side of (1) divided by $-2 \pi$. (The real part of the integral (2) may be found in standard tables.)

\section{The PaCkages}

The computer proof shown in the following section employs two packages both developed at RISC. First, we use Wegschaider's [7] MultiSum ${ }^{1}$ for computing recurrences for multiple hypergeometric sums. This Mathematica implementation extends the multivariate version of "Sister Celine's technique" developed by Wilf and Zeilberger [8]. For sake of brevity, we shall sketch the method only briefly here. For more details, the interested reader is referred to [7].

Let $\boldsymbol{j}=\left(j_{1}, \ldots, j_{s}\right)$ and $\boldsymbol{k}=\left(k_{1}, \ldots, k_{r}\right)$ be vectors of variables ranging over the integers. The central concept of the Sister Celine/WZ method is the computation of recurrences for multiple sums $\sum_{\boldsymbol{k}} f(\boldsymbol{j}, \boldsymbol{k})$, where $f(\boldsymbol{j}, \boldsymbol{k})$ is hypergeometric in all of its arguments. For this, one first computes a recurrence for the summand of the form

$$
\sum_{(\boldsymbol{a}, \boldsymbol{b}) \in S} \sigma_{\boldsymbol{a}, \boldsymbol{b}}(\boldsymbol{j}) f(\boldsymbol{j}+\boldsymbol{a}, \boldsymbol{k}+\boldsymbol{b})=0,
$$

where $S$, the structure set, is a finite set of integer tuples and the $\sigma_{\boldsymbol{a}, \boldsymbol{b}}(\boldsymbol{j})$ are polynomials not depending on $\boldsymbol{k}$ and not all zero. This turns out to be a very time- and memory-consuming task. In other words, in practice it succeeds only if the structure set, which is usually not known in advance, is chosen in a clever way. While

\footnotetext{
$1_{\text {available at http://www.risc.uni-linz.ac.at/research/combinat/risc/software/MultiSum }}$
} 
Wegschaider incorporated ideas of Verbaeten [6] to improve efficiency considerably, here we shall make use of a new method due to Riese and Zimmermann [5]. The main idea is to use random parameter substitutions to quickly rule out useless structure sets.

Another subtle, albeit important, point is that Wegschaider's algorithm first computes a recurrence whose coefficients $\sigma_{\boldsymbol{a}, \boldsymbol{b}}(\boldsymbol{j})$ may-contrary to the WZ method-depend on the summation parameters $\boldsymbol{k}$, too. However, by imposing additional constraints, such a recurrence may be brought into the form

$$
\sum_{(\boldsymbol{a}, \boldsymbol{b}) \in S^{\prime}} \sigma_{\boldsymbol{a}, \boldsymbol{b}}^{\prime}(\boldsymbol{j}) f(\boldsymbol{j}+\boldsymbol{a}, \boldsymbol{k}+\boldsymbol{b})=\Delta_{k_{1}}\left(f_{1}(\boldsymbol{j}, \boldsymbol{k})\right)+\cdots+\Delta_{k_{r}}\left(f_{r}(\boldsymbol{j}, \boldsymbol{k})\right),
$$

where $\Delta_{k}$ denotes the forward difference operator given by $\Delta_{k}(f(k))=f(k+1)-$ $f(k)$, the $f_{i}$ are certain linear combinations of shifts of the original summand $f$, and the $\sigma_{\boldsymbol{a}, \boldsymbol{b}}^{\prime}(\boldsymbol{j})$ are again free of $\boldsymbol{k}$. This recurrence now has the appropriate form for summation and immediately yields a recurrence for the whole sum.

In the second part of the proof, we shall compute closed forms for the inner sums of $S(1)$ and $S(2)$. This will be achieved with the Paule/Schorn ${ }^{2}$ implementation [3] of Zeilberger's fast algorithm [4], which computes recurrences for hypergeometric single sums $\sum_{k} f(n, k)$. This is done by first determining polynomials $\sigma_{i}(n)$ and a rational function $r(n, k)$ such that

$$
\sum_{i=0}^{d} \sigma_{i}(n) f(n+i, k)=\Delta_{k}(r(n, k) f(n, k)) .
$$

Again, this recurrence may be summed easily, leading to the recurrence for the sum.

In our situation, we shall be faced with one more aspect of Zeilberger's algorithm, namely, with the fact that it does not always deliver the recurrence of minimal order. Thus, by looking at the output, we would not be able to realize immediately that the inner sums of $S(1)$ and $S(2)$ have closed forms, because the algorithm does not find the corresponding recurrences of order 1 . However, as observed by Paule [2], this phenomenon may be cured in many instances by creative symmetrizing, i.e., by making use of the summand's symmetry. Suppose that

$$
\sum_{k} f(n, k)=\sum_{k} f(n, t(n, k)),
$$

for some integer linear function $t(n, k)$. Then we have that

$$
\sum_{k} f(n, k)=\frac{1}{2} \sum_{k}[f(n, k)+f(n, t(n, k))]=\frac{1}{2} \sum_{k} f(n, k)\left(1+\frac{f(n, t(n, k))}{f(n, k)}\right) .
$$

Now, if $f(n, t(n, k)) / f(n, k)$ is a rational function, Zeilberger's algorithm may be applied also to the sum on the right-hand side and usually finds the recurrence of minimal order.

\section{Computer Proof of Theorem 1}

Defining

$$
f_{\epsilon}(j ; n, k):=\frac{(2 n-2+\epsilon) !}{(2 n-1-k) ! k ! 2^{2 n-1}(k-j-n+1 / 2)},
$$

2 available at $h t t p: / / w w w . r i s c . u n i$-linz.ac.at/research/combinat/risc/software/PauleSchorn 
one obviously has that

$$
S(j)=\lim _{\epsilon \rightarrow 0} \sum_{n \geq 1} \sum_{k} f_{\epsilon}(j ; n, k) .
$$

The advantage of introducing the extra parameter $\epsilon$ in (3) is simply that the summand then vanishes for all $n<1$. (Note that due to the factorials involved, $f_{\epsilon}(j ; n, k)$ also vanishes for $k<0$ or $k>2 n-1$.)

3.1. The General Recurrence. In the first part of the proof, we derive a recurrence for $f_{\epsilon}(j ; n, k)$. For this, we start out by utilizing a brand new feature of MultiSum that quickly determines a small structure set.

$$
\begin{aligned}
& \operatorname{In}[1]:=<<\text { MultiSum.m } \\
& \text { MultiSum Package by Kurt Wegschaider - C RISC Linz }-\mathrm{V} 1.46 \beta(04 / 16 / 02) \\
& \operatorname{In}[2]:= f_{\epsilon}=(2 n-2+\epsilon) ! /\left((2 n-1-k) ! k ! 2^{2 n-1}(k-j-n+1 / 2)\right) \\
& \operatorname{In}[3]:= S=\text { FindStructureSet }\left[f_{\epsilon}, j, 2,\{n, k\},\{1,1\},\{2,0\}, \mathrm{WZ} \rightarrow\right. \text { True, } \\
&\text { Protocol } \rightarrow \text { False, NumericCheck } \rightarrow \text { True }][[1]] \\
& \text { Out[3]= }\{\{0,0,0\},\{0,1,0\},\{0,1,1\},\{1,0,0\},\{1,1,0\},\{1,1,1\},\{2,0,0\},\{2,1,1\}\}
\end{aligned}
$$

Now we use this set to compute a symbolic solution. This takes just a few seconds.

$$
\begin{aligned}
& \operatorname{In}[4]:=\text { FindRecurrence }\left[f_{\epsilon}, j,\{n, k\}, S,\{2,0\}, \text { WZ } \rightarrow \text { True, Protocol } \rightarrow \text { False }\right] \\
& \text { Out[4] }=(-4+2 j+\epsilon)(-3+2 j+\epsilon) F(-2+j,-1+n,-1+k)- \\
& 2\left(4-8 j+4 j^{2}+\epsilon+\epsilon^{2}\right) F(-1+j,-1+n,-1+k)+ \\
& (-1+2 j-\epsilon)(2 j-\epsilon) F(j,-1+n,-1+k)= \\
& \Delta_{k}(-(-1+2 j-2 n)(-7+2 j+2 n) F(-2+j,-1+n,-1+k)+ \\
& (-5+2 j+2 n)(1+4 j-4 n-2 \epsilon) F(-1+j,-1+n,-1+k)- \\
& 2(-3+2 j+2 n)(1+j-n-\epsilon) F(j,-1+n,-1+k))+ \\
& \Delta_{n}(-(-1+2 j-2 n)(-7+2 j+2 n) F(-2+j,-1+n, k))+ \\
& 2(1+2 j-2 n)(-5+2 j+2 n) F(-1+j,-1+n, k)- \\
& (3+2 j-2 n)(-3+2 j+2 n) F(j,-1+n, k))
\end{aligned}
$$

Next, we sum over the recurrence and set $\epsilon=0$ afterwards; see the remark below.

$$
\begin{aligned}
\ln [5]:= & \text { SumCertificate[\%] } / . \epsilon \rightarrow 0 \\
\text { Out[5]= } & (-4+2 j)(-3+2 j) \operatorname{SUM}(-2+j)-2\left(4-8 j+4 j^{2}\right) \operatorname{SUM}(-1+j)+ \\
& 2 j(-1+2 j) \operatorname{SUM}(j)=0
\end{aligned}
$$

Simplification yields:

$\operatorname{In}[6]:=$ ShiftRecurrence[\%, $\{j, 2\}] / /$ SimplifyRecurrence

$$
\begin{gathered}
\text { Out[6]= } 2 j(1+2 j) \operatorname{SUM}(j)-8(1+j)^{2} \operatorname{SUM}(1+j)+ \\
2(2+j)(3+2 j) \operatorname{SUM}(2+j)=0
\end{gathered}
$$

Finally, we plug in the right-hand side of (1) and obtain

$$
\begin{aligned}
\ln [7]:=\% / . & \operatorname{SUM}\left[j+a_{-}\right] \rightarrow \\
& j /(j+a) \operatorname{SUM}[j]+(-2 /(j+a)) \operatorname{Sum}[1 /(2 m-1),\{m, j+1, j+a\}]
\end{aligned}
$$




$$
\begin{gathered}
\text { Out[7]=2 } \\
j(1+2 j) \operatorname{SUM}(j)-8(1+j)^{2}\left(\frac{-2}{(1+j)(-1+2(1+j))}+\frac{j \operatorname{SUM}(j)}{1+j}\right)+ \\
2(2+j)(3+2 j)\left(\frac{-2\left(\frac{1}{-1+2(1+j)}+\frac{1}{-1+2(2+j)}\right)}{2+j}+\frac{j \operatorname{SUM}(j)}{2+j}\right)=0
\end{gathered}
$$

which, after built-in simplification, shows that it indeed satisfies the recurrence:

$$
\begin{aligned}
\ln [8] & =\text { Simplify[\%] } \\
\text { Out[8] } & =\text { True }
\end{aligned}
$$

Remark. There is no need to make the double sum terminate with respect to $n$. Just sum the recurrence obtained in Out[4] over $n \leq N$ and observe that as $N \rightarrow \infty$, the resulting terms on the right-hand side all vanish, because they are just polynomials $p_{i}(\epsilon, j, N, k)$ multiplied with $f_{\epsilon}\left(j-a_{i} ; N-b_{i}, k-c_{i}\right)$ and $N^{i} f_{\epsilon}(j ; N, k) \rightarrow 0$ as $N \rightarrow \infty$.

3.2. The Initial Values. It remains to prove that (1) holds for the initial values $j=1$ and $j=2$. Surprisingly, the inner sum of $S(j)$ has a closed form in both cases, which may be found by using Zeilberger's algorithm. As mentioned in Section 2, we need creative symmetrizing. Observe that

$$
\begin{aligned}
\sum_{k} f_{0}(j ; n, k) & =\frac{1}{2} \sum_{k}\left[f_{0}(j ; n, k)+f_{0}(j ; n, 2 n-1-k)\right] \\
& =\frac{1}{2} \sum_{k} f_{0}(j ; n, k)\left(1+\frac{k-n-j+1 / 2}{n-k-j-1 / 2}\right) .
\end{aligned}
$$

Now Zeilberger's algorithm applied to the symmetrized summand in fact computes a recurrence of order 1 (whereas it finds a recurrence only of order 2 for the unmodified summand).

$$
\begin{aligned}
\operatorname{In}[9]:= & <<\text { zb.m } \\
& \text { Fast Zeilberger Package by Peter Paule, Markus Schorn, and Axel Riese - } \\
& \text { C RISC Linz }-\mathrm{V} 3.30 \beta(03 / 21 / 02) \\
\operatorname{In}[10]:= & \text { symm- } \mathrm{f}_{0}=(1+(k-n-j+1 / 2) /(n-k-j-1 / 2)) f_{\epsilon} / . \epsilon \rightarrow 0 ; \\
\operatorname{In}[11]:= & \mathrm{Zb}\left[\text { symm- } \mathrm{f}_{0} / \cdot j \rightarrow 1,\{k, 0,2 n-1\}, n, 1\right] \\
& \text { If ' }-1+2 n \text { ' is a natural number, then: } \\
\text { Out[11] }= & \{(-1+2 n) \operatorname{SUM}(n)+(-3-2 n) \operatorname{SUM}(1+n)=0\} \\
\operatorname{In}[12]:= & \mathrm{Zb}\left[\operatorname{symm}-\mathrm{f}_{0} / \cdot j \rightarrow 2,\{k, 0,2 n-1\}, n, 1\right] \\
& \text { If ' }-1+2 n \text { ' is a natural number, then: } \\
\text { Out[12]= } & \{(1+2 n) \operatorname{SUM}(n)+(-5-2 n) \operatorname{SUM}(1+n)=0\}
\end{aligned}
$$

From this and the initial values for $n=1$, one gets that the inner sum equals

$$
\frac{-4}{(2 n-1)(2 n+1)} \text { and } \frac{-8}{(2 n+1)(2 n+3)},
$$

respectively. This completes the proof, because by partial fraction decomposition we immediately obtain

$$
\begin{aligned}
\operatorname{In}[13] & :=\operatorname{Sum}[-4 /((2 n-1)(2 n+1)),\{n, 1, \infty\}] \\
\text { Out }[13] & =-2
\end{aligned}
$$




$$
\begin{aligned}
\ln [13] & :=\operatorname{Sum}[-8 /((2 n+1)(2 n+3)),\{n, 1, \infty\}] \\
\text { Out[13] } & =-\frac{4}{3}
\end{aligned}
$$

which agree with the initial values for the right-hand side of (1).

Remark. Although one might get the impression from the cases $j=1$ and $j=2$ that there exists a hypergeometric closed form for general $j$, no such closed form for the inner sum is available.

\section{REFERENCES}

1. Lyons, R., Steif, J.: Stationary determinantal processes: Phase transitions, Bernoullicity, entropy, and domination. Preprint (2002)

2. Paule, P.: Short and easy computer proofs of the Rogers-Ramanujan identities and of identities of similar type. Electr. J. Combin. 1 (1994), \#R10

3. Paule, P., Schorn, M.: A Mathematica version of Zeilberger's algorithm for proving binomial coefficient identities. J. Symbolic Comput. 20, 673-698 (1995)

4. Petkovšek, M., Wilf, H.S., Zeilberger, D.: $A=B$. Wellesley: A.K. Peters 1996

5. Riese, A., Zimmermann, B.: Randomization speeds up hypergeometric summation. In preparation $(2002)$

6. Verbaeten, P.: Rekursiebetrekkingen voor lineaire hypergeometrische funkties. Ph.D. thesis, Katholieke Universiteit te Leuven, Heverlee, Belgium (1976)

7. Wegschaider, K.: Computer generated proofs of binomial multi-sum identities. Diploma thesis, RISC, J. Kepler University, Linz, Austria (1997)

8. Wilf, H.S., Zeilberger, D.: An algorithmic proof theory for hypergeometric (ordinary and "q") multisum/integral identities. Invent. Math. 108, 575-633 (1992)

9. Zeilberger, D.: A holonomic systems approach to special functions identities. J. Comput. Appl. Math. 32, 321-368 (1990)

Department of Mathematics, Indiana University, Bloomington, IN 47405-5701, USA and School of Mathematics, Georgia Institute of Technology, Atlanta, GA 30332-0160, USA

E-mail address: rdlyons@indiana.edu

Research Institute for Symbolic Computation, Johannes Kepler University, A-4040 Linz, Austria

E-mail address: Peter.Paule@risc.uni-linz.ac.at

Research Institute for Symbolic Computation, Johannes Kepler University, A-4040 LinZ, AUSTRIA

E-mail address: Axel.Riese@risc.uni-linz.ac.at 